Experiments and models of MHD jets and their relevance to astrophysics and solar physics

Paul M. Bellan

Citation: Physics of Plasmas 25, 055601 (2018); doi: 10.1063/1.5009571

View online: https://doi.org/10.1063/1.5009571

View Table of Contents: http://aip.scitation.org/toc/php/25/5

Published by the American Institute of Physics

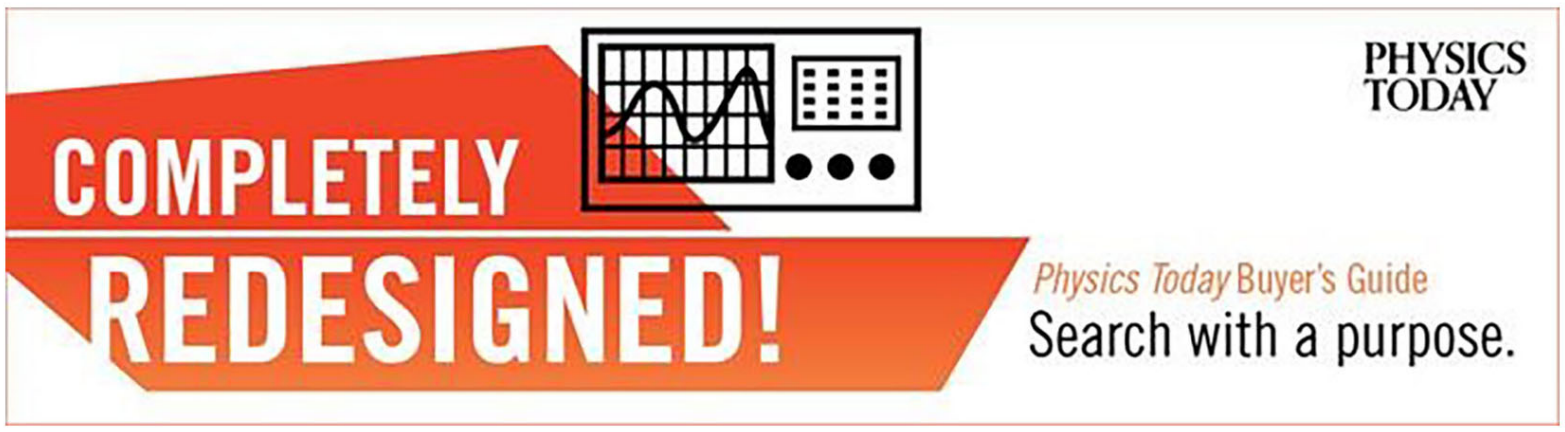




\title{
Experiments and models of MHD jets and their relevance to astrophysics and solar physics
}

\author{
Paul M. Bellan ${ }^{\text {a) }}$ \\ Applied Physics and Materials Science, California Institute of Technology, Pasadena, California 91125, USA
}

(Received 17 October 2017; accepted 7 December 2017; published online 12 March 2018)

\begin{abstract}
Magnetohydrodynamic (MHD)-driven jets involve poloidal and toroidal magnetic fields, finite pressure gradients, and unbalanced forces. The mechanism driving these jets is first discussed qualitatively by decomposing the magnetic force into a curvature and a gradient component. The mechanism is then considered quantitatively by consideration of all terms in the three components of the MHD equation of motion and in addition, the implications of Ampere's law, Faraday's law, the ideal Ohm's law, and the equation of continuity. The analysis shows that jets are self-collimating with the tip of the jet moving more slowly than the main column of the jet so there is a continuous stagnation near the tip in the jet frame. Experiments supporting these conclusions are discussed and it is shown how this mechanism relates to jets in astrophysical and solar corona contexts. Published by AIP Publishing. https://doi.org/10.1063/1.5009571
\end{abstract}

\section{INTRODUCTION}

This tutorial paper describes magnetohydrodynamic (MHD) jets that occur in laboratory experiments and in nature. Jet physics will be described in terms of basic MHD properties which will first be listed and then discussed; the discussion assumes that the reader has at least a minimal familiarity with the equations of ideal MHD. Naturally occurring MHD jets include astrophysical jets ${ }^{1-4}$ and aspects of solar corona loops. ${ }^{5}$ Laboratory examples include coaxial magnetized plasma guns used to make spheromaks ${ }^{6-8}$ and MHD thrusters being investigated for space propulsion. In contrast to Marshall guns ${ }^{9}$ and electric $\operatorname{arcs}^{10,11}$ which have a toroidal magnetic field but no poloidal field, the jets considered here have both toroidal and poloidal fields and so have magnetic helicity.

First observed a century ago, ${ }^{12}$ astrophysical jets are now known to exist over a wide range of scales with the smallest jets having lengths of the order of the size of the solar system and the largest being millions of parsecs long. ${ }^{13}$ There is large astrophysical literature on the theory of astrophysical jets with notable examples being Refs. 14-18 and during the last two decades most of this literature has involved numerical solutions of the MHD equations. However, there are also some analytical treatments, for example Ref. 19. Astrophysical jets can be either nonrelativistic or relativistic with the solar-system-size jets $\left(10^{-4}\right.$ parsec characteristic length) being non-relativistic and jets with kiloparsec-megaparsec characteristic lengths being highly relativistic. This paper will only consider nonrelativistic jets. The solar corona has a variety of plasmafilled magnetic flux tubes that are sometimes stationary and sometimes violently erupt. It will be argued here that MHD jet concepts apply to some of this solar behavior.

MHD jets have been studied in the laboratory using various technologies each having a distinctive parameter regime.

Note: Paper PT2 1, Bull. Am. Phys. Soc. 62, 261 (2017).

${ }^{a)}$ Invited speaker.
Z-pinch technology has been used at Imperial College by Lebedev et al. ${ }^{20}$ to create jets with a mm scale size and submicrosecond time scales. Laser technology has been used at the University of Rochester by Li et al. ${ }^{21}$ to make jets with megagauss magnetic fields and nanosecond characteristic times. Spheromak technology has been used at Caltech to make jets with kilogauss fields, time scales of tens of microseconds, and tens of $\mathrm{cm}$ characteristic lengths ${ }^{22-24}$ and this technology has also been used at Caltech to simulate solar coronal loops and show ${ }^{25,26}$ that MHD jets are an important aspect of these loops. MHD jets have also been investigated as the basis for spacecraft propulsion at the Consorzio RFX in Italy by Zuin $e t a l .{ }^{27}$ and at Princeton University by Lev and Choueiri. ${ }^{28}$

The above list of situations where MHD jets occur spans twenty orders of magnitude in time and space; yet, these situations have the same morphology, topology, and dynamical mechanism. The morphology is axisymmetric with a long, narrow, collimated jet, the topology involves linked poloidal and toroidal magnetic fields, and the dynamical mechanism is based on hydromagnetic forces.

\section{JETS COMPARED TO TRADITIONAL PLASMA STUDIES}

Traditional study of plasmas starts by considering infinite uniform plasmas and then the more realistic situation of a finite-dimension equilibrium configuration. Once an equilibrium has been established, study then focuses on unstable perturbations about this equilibrium or stable perturbations as constituted by various types of waves. Jets fall into none of the above categories because jets are not in equilibrium since there are unbalanced forces that accelerate the plasma and cause the dimensions to change with time. On the other hand, the qualitative morphology of jets remains constant even though the dimensions are changing so jets can be considered quasi-steady. Traditional studies of plasmas typically invoke various simplifying assumptions such as: (i) the 
magnetic field is constant in which case the electric field vanishes or is electrostatic, (ii) the plasma is incompressible, and (iii) magnetic flux, total mass, and total energy are all conserved. None of these assumptions hold for jets and the study of jets requires taking into account time-dependent magnetic fields, finite electric fields, compressibility, nonconstant mass, and non-constant magnetic flux. ${ }^{29,30}$ Jets are thus open systems where mass, flux, and energy are being continuously injected at a boundary. Despite the lack of conserved quantities, it is possible to develop a good understanding of how jets work because the jet axisymmetry greatly constrains the magnetic topology and enables a detailed analysis. We now list the various equations being used and then will show how a combination of well-known properties of these equations provides the basis for jet behavior.

\section{EQUATIONS AND MAGNETIC FIELD PROPERTIES}

The basic description of jets thus requires all of the following ideal MHD equations:

$$
\begin{gathered}
\rho\left(\frac{\partial \mathbf{U}}{\partial t}+\mathbf{U} \cdot \nabla \mathbf{U}\right)=\mathbf{J} \times \mathbf{B}-\nabla P, \\
\frac{\partial \mathbf{B}}{\partial t}=-\nabla \times \mathbf{E}, \\
\mathbf{E}+\mathbf{U} \times \mathbf{B}=0, \\
\nabla \times \mathbf{B}=\mu_{0} \mathbf{J}, \\
\frac{\partial \rho}{\partial t}+\nabla \cdot(\rho \mathbf{U})=0, \\
P \sim\left\{\begin{array}{c}
\rho^{\gamma}(\text { adiabatic }) \\
\rho(\text { isothermal })
\end{array}\right.
\end{gathered}
$$

these equations are the equation of motion, Faraday's law, the ideal MHD Ohm's law, Ampere's law, the continuity equation, and an equation of state, respectively.

These equations have no intrinsic scale and so can describe situations ranging from the laboratory to astrophysics and so, by changing the characteristic length and time, a laboratory result can be scaled to an astrophysical situation. ${ }^{31}$ Combination of Eqs. 1(b) and 1(c) gives the induction equation

$$
\frac{\partial \mathbf{B}}{\partial t}=\nabla \times(\mathbf{U} \times \mathbf{B})
$$

which shows that magnetic flux is frozen into the plasma frame. This is a strong statement because it means that the magnetic flux through an arbitrarily chosen surface $S(t)$ moving with the plasma remains constant.

A vacuum (also called potential) magnetic field is a magnetic field with no associated current density and so satisfies $\nabla \times \mathbf{B}=0$. The magnetic field energy is

$$
W=\int \frac{B^{2}}{2 \mu_{0}} d \mathbf{r}
$$

and it is straightforward to show (e.g., Sec. 9.2 of Ref. 29) that the vacuum magnetic field is the lowest energy magnetic field for given boundary conditions. This means that if a magnetic field is initially a vacuum field but then is altered without changing the boundary conditions, more energy is stored in the altered field than was in the original form. Thus, it takes work to deform a magnetic field away from its vacuum state if the boundary conditions are held fixed. One can think of the magnetic field as being "elastic" in such a way that it "wants" to revert to its vacuum state.

Traditional studies of MHD equilibria have been based on an increasingly complex hierarchy of approximations as follows:

1. Potential (vacuum) magnetic field: $\mathbf{E}, \mathbf{U}, \mathbf{J}$, and $\nabla P$ in Eqs. 1(a)-1(e) are assumed to be zero and $\rho$ is assumed to be spatially uniform. The magnetic field $\mathbf{B}$ is assumed to be the gradient of a potential, i.e., $\mathbf{B}=\nabla \chi$ so $\nabla \times \mathbf{B}=0$. The potential approximation has often been used to model the solar corona magnetic field. ${ }^{32,33}$

2. Linear force-free magnetic field: $\mathbf{E}, \mathbf{U}$, and $\nabla P$ in Eqs. 1(a)-1(e) are assumed to be zero and $\rho$ is assumed to be spatially uniform. The magnetic field and the current density $\mathbf{J}$ are non-zero but are such that $\mu_{0} \mathbf{J}=\lambda \mathbf{B}$ where $\lambda$ is spatially uniform. The Woltjer-Taylor relaxation theory ${ }^{34-37}$ predicts that a low $\beta$ plasma will self-organize to this state which is called "force-free" because $\mathbf{J} \times \mathbf{B}=0$. The simplest force-free situation is the Lundquist solution $^{38}$ which assumes cylindrical geometry and has $B_{z}$ $=B_{0} J_{0}(\lambda r)$ and $B_{\phi}=B_{0} J_{1}(\lambda r)$, where $J_{0}$ and $J_{1}$ are Bessel's functions and $B_{0}$ is a constant. The linear forcefree field is the basis of spheromaks ${ }^{6-8}$ and reverse field pinches ${ }^{36}$ and has also been used to characterize the solar corona. $^{39,40}$

3. Non-linear force-free field: This is the same as force-free except now $\lambda$ is no longer assumed to be spatially uniform. However, the $\nabla \cdot \mathbf{J}=0$ requirement constrains $\lambda$ to be constant along a magnetic field line since $\nabla \cdot \mathbf{J}$ $=\nabla \cdot\left(\lambda \mathbf{B} / \mu_{0}\right)=0$ implies $\mathbf{B} \cdot \nabla \lambda=0$. The non-linear force-free field has been used in numerical models of the solar corona. ${ }^{41}$

4. Bennett pinch: ${ }^{42} \mathbf{E}$ and $\mathbf{U}$ in Eqs. 1(a)-1(e) are assumed to be zero, while $P$ and $\rho$ are allowed to be finite and spatially non-uniform. Cylindrical geometry with azimuthal symmetry is assumed and furthermore, the configuration is assumed to be translationally invariant in the $z$ direction and thus infinite in the $z$ direction. Unlike the potential field and force-free equilibria, here a finite pressure gradient is assumed so now $\mathbf{B}, \mathbf{J}$, and $\nabla P$ are all finite. Equation 1(a) reduces to

$$
\mathbf{J} \times \mathbf{B}=\nabla P .
$$

There is neither a pressure gradient nor a magnetic force in each of the $z$ and $\phi$ directions as both $z$ and $\phi$ are ignorable coordinates. Thus, all quantities depend on $r$ only. The equilibrium is determined by solving for radial force balance, i.e., by solving $J_{\phi} B_{z}-J_{z} B_{\phi}=\partial P / \partial r$. There is no current or magnetic field in the $r$ direction as stipulated 
by $\nabla \cdot \mathbf{B}=0$ and $\nabla \cdot \mathbf{J}=0$. If the current is only in the $z$-direction, the configuration is called a Z-pinch and if the current is only in the $\phi$-direction, the configuration is called a theta-pinch ( $\theta$ rather than $\phi$ is typically used to denote the azimuthal coordinate in this situation).

5. Grad-Shafranov equation: ${ }^{43,44} \mathbf{E}$ and $\mathbf{U}$ are assumed to be zero. Cylindrical geometry with azimuthal symmetry is assumed, but unlike the Bennett pinch, the configuration now depends on both $r$ and $z$ and the pressure is finite in a localized, finite-dimension 3D volume. The configuration is governed by Eq. (4) and all three components $B_{r}, B_{\phi}$ and $B_{z}$ of the magnetic field and all three components $J_{r}, J_{\phi}$, and $J_{z}$ of the current density are finite. The Grad-Shafranov equation is used to describe tokamaks $^{45}$ as well as interplanetary magnetic clouds ${ }^{46}$ resulting from eruptions of structures on the solar corona.

It is sometimes presumed that the MHD force balance given by Eq. (4) can be satisfied even if there is no symmetry; i.e., it is sometimes assumed that given some arbitrary magnetic field $\mathbf{B}(\mathbf{r})$, it should be possible to find a pressure profile $P(\mathbf{r})$ that satisfies Eq. (4) with $\mathbf{J}$ given by Eq. 1(d). This presumption is typically invoked by sketching an arbitrary magnetic field line configuration and then assuming that the sketched field represents an MHD equilibrium. However, this presumption is incorrect because (i) the curl of $\nabla P$ always vanishes because of a mathematical identity whereas (ii) there is no particular reason why the curl of $\mathbf{J}$ $\times \mathbf{B}$ should be zero. For an arbitrary $\mathbf{B}(\mathbf{r})$, the curl of $\mathbf{J} \times \mathbf{B}$ is in fact typically finite. Thus, for an arbitrary magnetic field the right hand side of Eq. 1(a) is typically finite in which case there is a net force on the plasma. A net force implies that the plasma will be accelerated so $\mathbf{U}$ will be finite. If $\mathbf{U}$ is finite then Ohm's law, Eq. 1(c), shows that $\mathbf{E}$ will typically be finite and then Faraday's law shows that $\mathbf{B}$ will typically become time-dependent. All these considerations come into play when considering MHD jets and so jets can be considered as a non-equilibrium extension of the hierarchy of equilibria listed above. Although jets are not in equilibria, they can be considered quasi-steady in the sense that even though parameters are changing, the morphology and nature of the dynamics remain the same.

A good starting point for consideration of jets is a detailed examination of the magnetic force $\mathbf{J} \times \mathbf{B}$. This force can be interpreted in several different, but equivalent, ways each having advantages and disadvantages. The first way is simply to note that existence of a current $\mathbf{J}$ is obviously required to have a $\mathbf{J} \times \mathbf{B}$ force. This is useful because magnetic fields typically permeate all of space as magnetic nulls are rare, whereas, in contrast, currents are typically localized. To the extent that there are no magnetic nulls, the magnetic force is thus localized to regions where the current is finite.

On using Ampere's law to eliminate $\mathbf{J}$ in $\mathbf{J} \times \mathbf{B}$, the magnetic force can be interpreted as solely dependent on magnetic field properties. Invoking the vector identity $\nabla B^{2} / 2=\mathbf{B} \cdot \nabla \mathbf{B}+\mathbf{B} \times \nabla \times \mathbf{B}$ shows that the magnetic force can be decomposed as

$$
\begin{aligned}
\mu_{0} \mathbf{J} \times \mathbf{B} & =(\nabla \times \mathbf{B}) \times \mathbf{B} \\
& =\mathbf{B} \cdot \nabla \mathbf{B}-\nabla\left(\frac{B^{2}}{2}\right) \\
& =B \hat{B} \cdot \nabla(B \hat{B})-\nabla\left(\frac{B^{2}}{2}\right) \\
& =B \hat{B} \cdot \nabla \hat{B}+\hat{B} \hat{B} \cdot \nabla\left(\frac{B^{2}}{2}\right)-\nabla\left(\frac{B^{2}}{2}\right) \\
& =-B^{2} \frac{\hat{R}}{R}-\nabla_{\perp}\left(\frac{B^{2}}{2}\right),
\end{aligned}
$$

where in the last line $R$ is the local radius of curvature of the magnetic field and $\hat{R}$ is a unit vector pointing away from the center of curvature along this radius of curvature. These curvature relations can be understood by defining a local cylindrical coordinate system $\{R, \theta, Z\}$ with the $Z$-axis at the center of curvature of the magnetic field. Thus, by definition $\hat{B}=\hat{\theta}$ and the cylindrical unit vector relation $\hat{\theta} \cdot \nabla \hat{\theta}$ $=-\hat{R} / R$ gives the result $\hat{B} \cdot \nabla \hat{B}=-\hat{R} / R$ on replacing $\hat{\theta} \rightarrow \hat{B}$. We call the first term on the last line of Eq. (5) the curvature force and the second term the gradient force. The gradient force acts like a pressure gradient but only in the direction perpendicular to the magnetic field as denoted by the $\nabla_{\perp}$ operator. The last line in Eq. (5) is thus a "fieldonly" representation of the magnetic force. It must be realized that the curvature and gradient forces are not orthogonal to each other in general and in the particular case of a vacuum magnetic field (i.e., a field where $\mathbf{J}=0$ ), the curvature and gradient force are equal and opposite. The effect of the curvature force is to try to straighten out or reduce curvature, while the effect of the gradient force is to push from regions of large $B^{2}$ to small $B^{2}$ but only in the direction perpendicular to the local magnetic field.

Jets will be assumed to be axisymmetric, but no other simplifying assumptions will be made; thus, jets are a generalization of the Grad-Shafranov situation. Cylindrical coordinates $\{r, \phi, z\}$ will be used where the $r$ direction is called the radial direction, the $\phi$ direction is called the toroidal direction, and the $z$ direction is called the axial direction. We note that $\nabla \phi$ $=\hat{\phi} / r$ and whenever possible find it convenient to express vectors in terms of $\nabla \phi$ because the identity $\nabla \times \nabla \phi=0$ simplifies manipulations. If vectors were instead expressed in terms of $\hat{\phi}$ then, when taking the curl, one would have to keep track of distracting terms of the form $\nabla \times \hat{\phi}=\nabla \times(r \nabla \phi)$ $=\hat{r} \times \hat{\phi} / r=\hat{z} / r$ that eventually cancel.

Another important property of axisymmetry is the requirement that all physical parameters must be mathematically regular, i.e., must be analytical and so have finite spatial derivatives of all order. The regularity constraint implies ${ }^{47}$ that the $r$ and $\phi$ components of an axisymmetric physical vector must be an odd function of $r$, while the $z$ component of an axisymmetric vector and all axisymmetric scalars must be even functions of $r$. Being odd functions of $r$, the vector components $B_{r}, B_{\phi}, E_{r}, E_{\phi}, J_{r}, J_{\phi}, U_{r}, U_{\phi}$ must all vanish on the $z$ axis no matter what equations are used, whereas $B_{z}, E_{z}, J_{z}, U_{z}$, and $P$ can be finite on the $z$ axis and, if finite, must be even functions of $r$. 
Because of the assumed axisymmetry, all vectors can be decomposed into a poloidal component and a toroidal component. The toroidal component is in the $\phi$ direction, while the poloidal component is in some combination of the $r$ and $z$ directions. Thus, a toroidal vector is of the form $\mathbf{T}$ $=T \hat{\phi}$ while a poloidal vector is of the form $\mathbf{P}=P_{r} \hat{r}+P_{z} \hat{z}$. An axisymmetric magnetic field can therefore always be expressed as

$$
\mathbf{B}=\mathbf{B}_{p o l}+\mathbf{B}_{t o r},
$$

where the poloidal part of the magnetic field is

$$
\mathbf{B}_{p o l}=\frac{1}{2 \pi} \nabla \psi \times \nabla \phi
$$

and the toroidal part of the magnetic field is

$$
\mathbf{B}_{\text {tor }}=\frac{\mu_{0} I}{2 \pi} \nabla \phi .
$$

The magnetic field then depends on two scalar parameters, the poloidal flux $\psi(\mathbf{r}, t)$ and the poloidal current $I(\mathbf{r}, t)$. The poloidal flux is the magnetic flux linked by a circle around the $z$ axis having axial position $z$ and radius $r$; i.e.,

$$
\begin{aligned}
\int \mathbf{B}_{p o l} \cdot d \mathbf{s} & =\int_{0}^{r} \frac{1}{2 \pi} \nabla \psi \times \nabla \phi \cdot \hat{z} 2 \pi r d r \\
& =\int_{0}^{r} \frac{1}{2 \pi} \hat{r} \frac{\partial \psi}{\partial r} \times \frac{\hat{\phi}}{r} \cdot \hat{z} 2 \pi r d r=\psi .
\end{aligned}
$$

The poloidal current is the electric current linked by a circle around the $z$ axis having axial position $z$ and radius $r$ and a relation paralleling Eq. (9) relates the poloidal current density $\mathbf{J}_{p o l}$ to the poloidal current, i.e., $I=\int \mathbf{J}_{p o l} \cdot d \mathbf{s}$. Poloidal fields are produced by toroidal currents and vice versa. The forms of Eqs. (7) and (8) automatically satisfy $\nabla \cdot \mathbf{B}=0$.

An important property of Eq. (5) relevant to jets is that the magnetic force depends on the gradient and curvature of the magnetic field but depends on the magnitude only if there is a gradient or curvature. Thus, it is possible for the force associated with the toroidal part of the magnetic field to be comparable to or much stronger than the force associated with the poloidal part of the magnetic field even though the magnitude of the poloidal part greatly exceeds the magnitude of the toroidal part. An example occurs in the force-free Lundquist solution where the radially inward force from $B_{\phi}$ balances the radially outward force from $B_{z}$ even though at small radius $B_{z}=B_{0} J_{0}(\lambda r)$ greatly exceeds $B_{\phi}=B_{0} J_{1}(\lambda r)$.

\section{QUALITATIVE DESCRIPTION OF JETS}

Figure 1 sketches the basic components of a jet. There is cylindrical symmetry about the $z$ axis and we are primarily concerned with behavior in the upper half $r-z$ plane (positive $z$ region). Boundary conditions for this upper half-plane are imposed at $z=0$. In the case of a lab experiment, $z=0$ would correspond to the electrodes, while in an astrophysical jet situation $z=0$ would correspond to an accretion disk. It is assumed that the upper half plane (i.e., $z>0$ ) is governed by

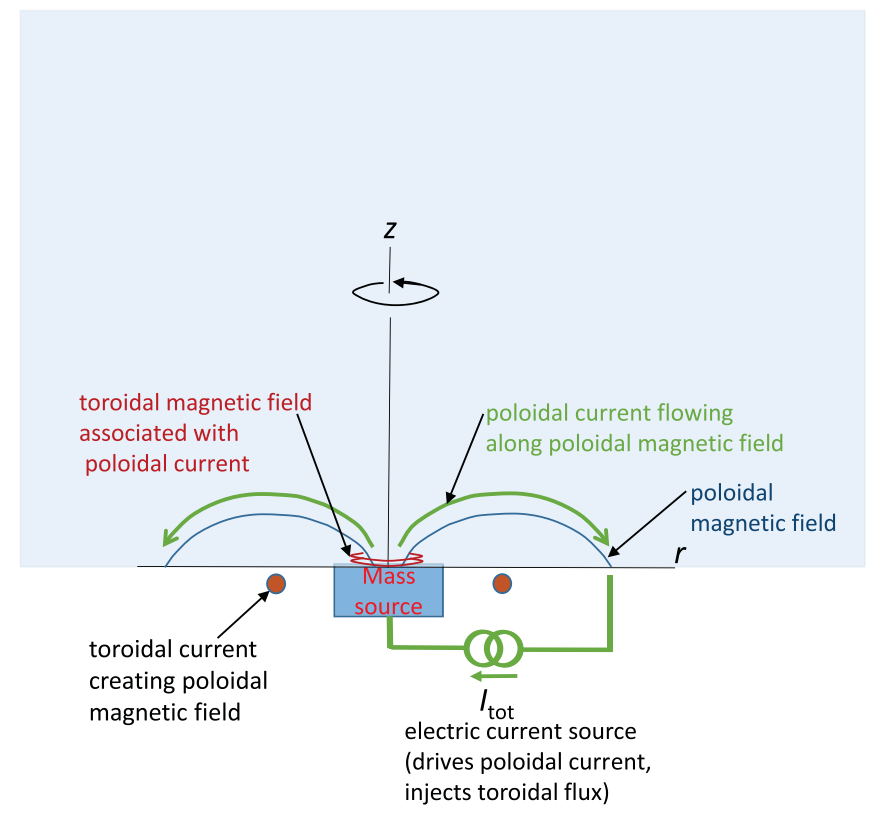

FIG. 1. Initial layout. Slight blue shading in the upper half plane $(z>0$ region) indicates initial diffuse plasma so that ideal MHD is valid.

ideal MHD but there is no such assumption for $z=0$ (i.e., it is not assumed that the electrodes or an accretion disk are governed by ideal MHD). The boundary conditions at $z=0$ are

1. A toroidal current located at or immediately below $z=0$ creates a dipole-like poloidal magnetic field in the upper half plane ( $z>0$ region).

2. A mass source at $z=0$ in the vicinity of the $z$-axis supplies an upward mass flux; the injection velocity is small compared to the eventual jet velocity.

3. There is initially sufficient diffuse plasma in the upper half $r-z$ plane so that this upper half plane is governed by ideal MHD. However, the density of this diffuse plasma is much lower than that of the mass source described in item 2 above.

4. A current source located below $z=0$ drives a poloidal electric current $I_{t o t}$ that flows in the upper half plane. The current source could be a battery, capacitor, or other voltage sources with a large resistance in series so the current flowing in the upper half-plane remains constant even if the plasma impedance changes. This current produces a toroidal magnetic field with toroidal flux frozen into the frame of the plasma. If plasma flows upwards from the mass source, then flux is frozen into this flow. As well as acting as a source of toroidal magnetic flux, the current source acts as a power source in accordance with Poynting's theorem. The dynamics causes a radial voltage drop to develop in the $z=0$ plane and the injected power is the product of this voltage and the electric current. The plasma has low impedance compared to the high internal impedance current source so the plasma looks like nearly a short-circuit to the current source.

The initial poloidal magnetic field is a vacuum field in the upper half $r-z$ plane since the toroidal current creating this field is not in the upper half $r-z$ plane. Thus, no forces 
are associated with the initial, undeformed poloidal magnetic field. Furthermore, being a vacuum magnetic field, the initial, undeformed poloidal magnetic field is a minimumenergy state.

In contrast, the toroidal field in Fig. 1 is not a vacuum field because the poloidal current that produces this field is in the upper half $r-z$ plane. The toroidal field is thus not in a minimum energy state and so there will be forces associated with this field. We first consider forces associated with a single toroidal field line and then forces associated with multiple toroidal field lines.

Since a single toroidal field line is a circle, there will be a magnetic curvature force $-\mu_{0}^{-1} B^{2} \hat{R} / R$ trying to contract the radius of this circle. There will also be a magnetic gradient force $-\mu_{0}^{-1} \nabla_{\perp}\left(B^{2} / 2\right)$ going from where the field is strong to where it is weak. The toroidal magnetic field must vanish on the $z$ axis (i.e., at $r=0$ ) because of mathematical regularity. ${ }^{47}$ The analysis in Ref. 47 shows that $B_{\phi}$ must scale as an odd power of $r$, so the toroidal field magnitude must initially increase with increasing $r$. This means that the gradient force is towards the $z$ axis, i.e., towards the center of curvature of the toroidal field. Thus, the curvature and gradient forces are both radially inwards and so toroidal field lines can be considered in this instance as behaving like elastic bands wrapped around the poloidal field and squeezing the poloidal field towards the $z$ axis. This is the pinch force.

Now consider the vertical behavior. It is seen in Fig. 1 that because the poloidal magnetic field flares radially outwards with increasing $z$ and because the poloidal current flows along the poloidal field, the current channel must also flare outwards (i.e., becomes wider with increasing height). This means that the current density decreases with increasing $z$. If $a(z)$ is the $z$-dependent radius of the current channel and $J_{z}$ is radially uniform, then $J_{z}=I_{t o t} /\left(\pi[a(z)]^{2}\right)$ and $B_{\phi}(r, z)=\mu_{0} r J_{z} / 2=\mu_{0} r I_{t o t} /\left(2 \pi[a(z)]^{2}\right)$. Thus, $B_{\phi}^{2}$ decreases with increasing $z$ at fixed $r$ because the widening of the current channel with height means that $a$ is an increasing function of $z$. Since the gradient magnetic force is directed from a high magnitude field region to a low magnitude field region, there will be an upward magnetic gradient force scaling as $-\partial B_{\phi}^{2} / \partial z$, i.e., proportional to $d a / d z$. Thus, the combination of magnetic curvature and magnetic gradient forces gives radially inward and axially upward forces as indicated in Fig. 2.

We now recall that magnetic flux is frozen into the frame of the plasma. This is true separately for the poloidal and toroidal fluxes because the mathematical proof for frozen-in flux allows the contour defining the perimeter of the flux to be arbitrarily chosen (see Sec. 2.6.4 of Ref. 29). Thus, one could choose a contour linking poloidal flux or one could choose a contour linking toroidal flux and in either case the linked flux would be conserved provided the contour moved with the plasma. The pinch force resulting from the sum of the curvature and radial gradient of the toroidal magnetic field squeezes the plasma radially. Because the poloidal magnetic field is frozen into the frame of the plasma, this force must also squeeze the poloidal magnetic field lines radially towards the $z$ axis. Similarly, the upwards axial force resulting from the axial gradient of the toroidal field

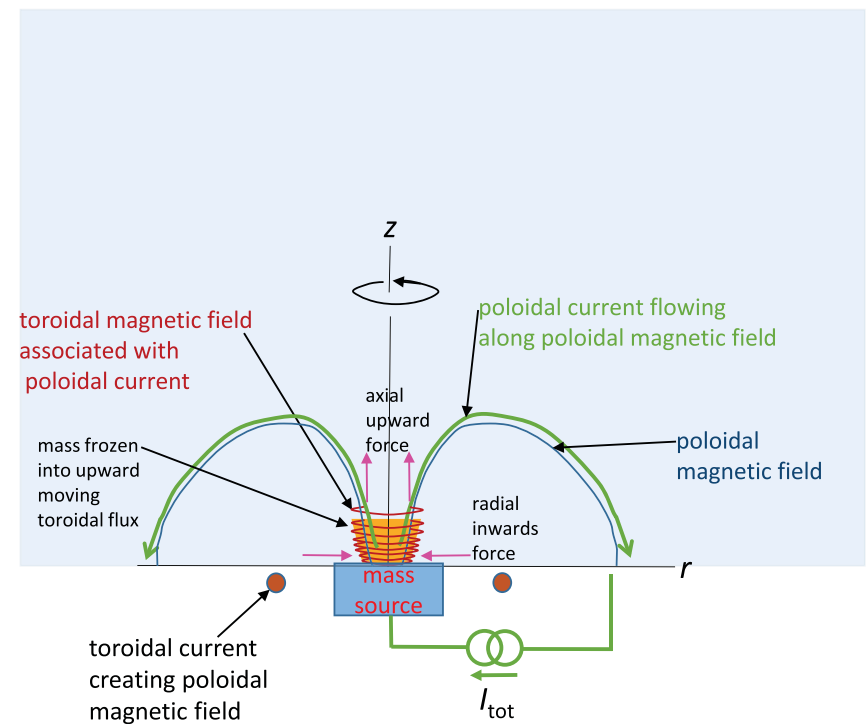

FIG. 2. Jet soon after beginning. Radial inward force from toroidal field curvature and gradient squeezes poloidal field and frozen-in plasma at small $z$. Axial upwards force from the gradient of toroidal field in the $z$ direction accelerates plasma from the $z=0$ region upwards with its frozen-in toroidal flux.

magnitude pushes plasma axially upwards, but because the poloidal field is frozen into the plasma, the poloidal field is also pushed axially upwards.

The poloidal field line length increases on being stretched. However, the poloidal current was assumed to remain constant because this current is being driven by a current source. There thus must be new toroidal flux entering the upper half plane from $z=0$ because toroidal flux is frozen into the plasma which is moving up from $z=0$. This injection of toroidal flux at $z=0$ is consistent with Ampere's law because, as the poloidal field stretches and becomes longer, more toroidal field lines link the poloidal field. There is consequently injection of both toroidal flux and mass. The rate of toroidal flux injection gives the voltage appearing across the current source, while the rate of mass injection is determined by the properties of the mass source. If the mass source is removed, the jet will become mass-starved and will not function.

This mass upflow and associated frozen-in magnetic field can be considered as constituting the jet. As seen in Fig. 3 , the axial stretching of the poloidal magnetic field combined with the simultaneous radially inward compression of the inner part of this field causes the radially inward part of the poloidal field in the jet main column to become nearly straight. The poloidal magnetic field curvature thus concentrates at the apex of the arched poloidal field (tip region in sketch) and this curvature provides a localized downward retarding force. This retarding force slows down the jet as it approaches large $z$. An observer located just before the jet tip and moving with the jet will see the jet material in front (higher $z$ location in sketch) moving more slowly than the jet material behind (lower $z$ location). This means that there will be an effective axial compression of the jet material in the observer's frame. This axial plasma compression also provides an axial compression of the frozen-in toroidal flux. 


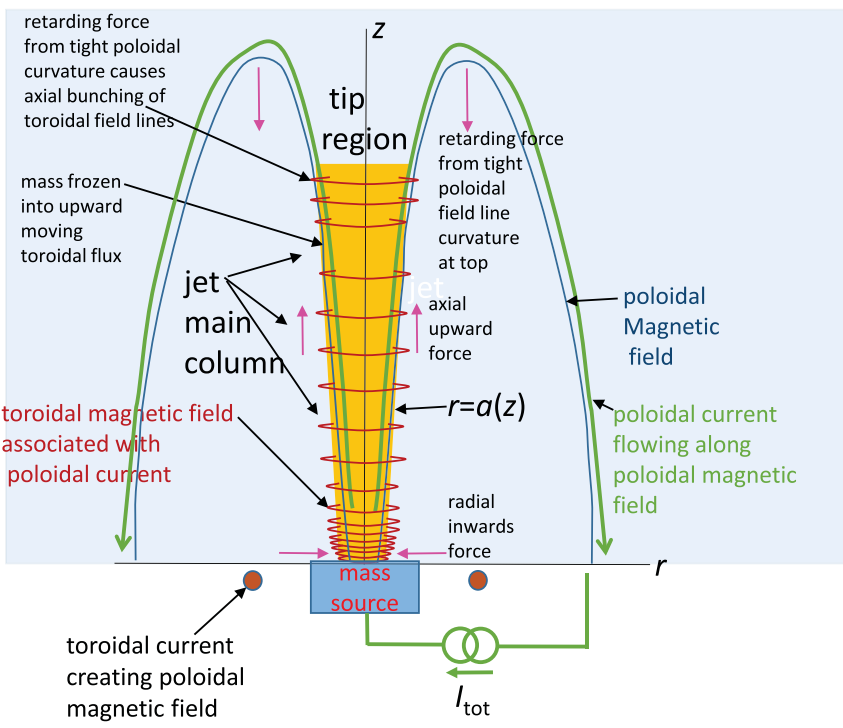

FIG. 3. Jet at the later stage. The stretching of the poloidal field causes the poloidal field to be nearly straight except at the apex where it makes a sharp turn-around. This sharp curvature at the top provides a downwards or retarding force that slows down the jet near its upper tip. The velocity gradient provides an axial compression in the jet frame of the jet plasma and its embedded toroidal flux. This compression increases the toroidal flux density, i.e., amplifies the toroidal field. The radially inward curvature and gradient forces of the amplified toroidal field collimate the jet.

However, since toroidal flux density corresponds to $B_{\phi}$, the axial squeezing increases $B_{\phi}$ and so increases the radial inward pinch force associated with the curvature and gradient of the toroidal field. The result is that the jet radius is reduced and so the jet self-collimates as it propagates. This process can continue forever so an arbitrarily long collimated jet results if no instabilities occur.

The upward magnetic force resulting from toroidal field curvature and gradient vanishes at $r=0$ because the toroidal field vanishes as $r=0$. However, because there is a higher pressure on the $z$-axis at small $z$ compared to large $z$ there is also an upwards axial acceleration at $r=0$ because of this axial pressure gradient.

\section{QUANTITATIVE DESCRIPTION OF JETS}

We now validate the qualitative description in Sec. IV by making a detailed analysis of the ideal MHD equations with boundary conditions (i.e., mass source, current source at $z=0$, initial vacuum poloidal field) as shown in Figs. 1-3. Because the curl of a toroidal vector is poloidal and vice versa, Eq. 1(d) shows that the poloidal and toroidal current densities are

$$
\mathbf{J}_{p o l}=\frac{1}{2 \pi} \nabla I \times \nabla \phi
$$

and

$$
\mathbf{J}_{\text {tor }}=-\frac{r^{2}}{2 \pi \mu_{0}}\left[\nabla \cdot\left(\frac{1}{r^{2}} \nabla \psi\right)\right] \nabla \phi
$$

so

$$
\mathbf{J}=\mathbf{J}_{\text {pol }}+\mathbf{J}_{\text {tor }}
$$

Equation (10) is formally like Eq. (7), so $I(r, z, t)$ is formally like $\psi(r, z, t)$.

It is useful at this point in the discussion to consider the condition for electric current to flow from one poloidal flux surface to another. For this to happen the current density $\mathbf{J}$ would have to contain a component normal to the poloidal flux surface. Since $\nabla \psi$ is normal to poloidal flux surfaces, it would thus be necessary to have finite $\mathbf{J} \cdot \nabla \psi$. Since $\mathbf{J} \cdot \nabla \psi$ $=\mathbf{J}_{\text {pol }} \cdot \nabla \psi=(2 \pi)^{-1} \nabla I \times \nabla \phi \cdot \nabla \psi=-(2 \pi)^{-1} \nabla I \times \nabla \psi$ $\cdot \nabla \phi$, this means it would be necessary to have finite $\nabla I$ $\times \nabla \psi$ to have electric current flow across flux surfaces. If $I=I(\psi)$ so $\nabla I \times \nabla \psi=(\partial I / \partial \psi) \nabla \psi \times \nabla \psi=0$ then electric current would be constrained to flow on poloidal flux surfaces but not across these surfaces. The poloidal flux surfaces could then be visualized as being like the nested skins of an onion with these skins electrically insulated from each other. The condition for electric current to flow from one flux surface to another is therefore that $I$ is not a function of $\psi$.

We now combine Eqs. 1(a), 1(d), and 1(e) as follows: We add $U_{r}$ times Eq. 1(e) to the LHS of the $r$ component of Eq. 1(a), $r U_{\phi}$ times Eq. 1(e) to the LHS of $r$ times the $\phi$ component of Eq. 1(a), and $U_{z}$ times Eq. 1(e) to the LHS of the $z$ component of Eq. 1(a). We also substitute for $\mathbf{J}$ using Eq. (12). Taking into account that

$$
\begin{array}{r}
(\mathbf{U} \cdot \nabla \mathbf{U})_{r}=U_{r} \frac{\partial U_{r}}{\partial r}+U_{z} \frac{\partial U_{r}}{\partial z}-\frac{U_{\phi}^{2}}{r}, \\
(\mathbf{U} \cdot \nabla \mathbf{U})_{\phi}=U_{r} \frac{\partial U_{\phi}}{\partial r}+U_{z} \frac{\partial U_{\phi}}{\partial z}+\frac{U_{\phi} U_{r}}{r}, \\
(\mathbf{U} \cdot \nabla \mathbf{U})_{z}=U_{r} \frac{\partial U_{z}}{\partial r}+U_{z} \frac{\partial U_{z}}{\partial z}
\end{array}
$$

the $r, \phi$, and $z$ components of the equation of motion become, respectively,

$$
\begin{aligned}
& \frac{\partial}{\partial t}\left(\rho U_{r}\right)+\nabla \cdot\left(\rho U_{r} \mathbf{U}\right) \\
& =\frac{1}{4 \pi^{2}}\left(-\frac{1}{\mu_{0}} \frac{\partial \psi}{\partial r} \nabla \cdot\left(\frac{1}{r^{2}} \nabla \psi\right)-\frac{\mu_{0} I}{r^{2}} \frac{\partial I}{\partial r}\right)-\frac{\partial P}{\partial r}+\frac{\rho U_{\phi}^{2}}{r} \\
& \frac{\partial}{\partial t}\left(\rho r U_{\phi}\right)+\nabla \cdot\left(\rho r U_{\phi} \mathbf{U}\right)=\frac{1}{4 \pi^{2}}(\nabla I \times \nabla \psi \cdot \nabla \phi), \quad(14 \mathrm{~b}) \\
& \frac{\partial}{\partial t}\left(\rho U_{z}\right)+\nabla \cdot\left(\rho U_{z} \mathbf{U}\right) \\
& \quad=\frac{1}{4 \pi^{2}}\left(-\frac{1}{\mu_{0}} \frac{\partial \psi}{\partial z} \nabla \cdot\left(\frac{1}{r^{2}} \nabla \psi\right)-\frac{\mu_{0} I}{r^{2}} \frac{\partial I}{\partial z}\right)-\frac{\partial P}{\partial z} \cdot(14 \mathrm{c})
\end{aligned}
$$

Since no approximations have been made, Eq. (14) is completely equivalent to Eq. 1(a).

We note in passing that the Grad-Shafranov equation is a reduced version of Eq. (1a) obtained by assuming $\mathbf{U}=0$ and $I=I(\psi)$. These assumptions give $P=P(\psi)$ so relations of the form $\partial I / \partial s=(\partial I / \partial \psi)(\partial \psi / \partial s)$ are obtained where $s=r, z$ and similarly for $\partial P / \partial s$. In this case, the RHS of both Eqs. 14(a) and 14(c) reduce to 


$$
\frac{1}{4 \pi^{2}}\left(\frac{1}{\mu_{0}} \nabla \cdot\left(\frac{1}{r^{2}} \nabla \psi\right)+\frac{\mu_{0} I}{r^{2}} \frac{\partial I}{\partial \psi}\right)+\frac{\partial P}{\partial \psi}=0,
$$

which is the Grad-Shafranov equation.

Jet behavior (i.e., finite $\mathbf{U}$ and no equilibrium) can be understood by examination of two different limiting approximations for Eq. (14). These relate, respectively, to the main jet column and the tip (leading edge) as labeled in Fig. 3 and also in the lab photo shown in Fig. 4. The main jet column is approximated as having a poloidal field that is nearly in the $z$ direction except for a slight radial flaring. The tip or leading edge is at the top in Figs. 3 and 4 and is characterized by having a strong curvature of the poloidal field. This curvature is unavoidable because the $z$ direction of the poloidal field reverses sign in this region. It will be shown in Sec. VII that the constant-current assumption implies $U_{\phi}=0$. Thus, the jet does not rotate and its lengthening helical magnetic field can be visualized as being like a machine screw being pushed through a clearance hole without rotating.

\section{A. Topology of jet poloidal flux}

The poloidal flux of a magnetic dipole located at the origin scales as $r^{2} /\left(r^{2}+z^{2}\right)^{3 / 2}$ and so is singular at the origin. However, by locating the dipole at $z=-h$ so the flux is now of the form

$$
\psi_{\text {dipole }}=\frac{r^{2}}{\left(r^{2}+(z+h)^{2}\right)^{3 / 2}}
$$

the poloidal flux is no longer singular at the origin and has the topology sketched in Figs. 1-4. This flux is zero both on the $z$ axis and at infinity in the upper half $r-z$ plane and has a maximum in the $z=0$ plane at $r=\sqrt{2} h$. The dipole poloidal flux corresponds to a vacuum field, i.e., substitution of Eq. (16) into Eq. (11) gives zero toroidal current. The jet poloidal flux is fixed in the $z=0$ plane and so could be set in this plane to be like that of a dipole. However, because of the stretching of the poloidal flux in the $z>0$ region, the jet poloidal flux differs from that of a dipole and so, no longer being a vacuum field, will have an associated finite toroidal current. Thus, the jet poloidal flux can be considered to scale as

$$
\psi \sim \frac{r^{2}}{\left(r^{2}+(z \sigma(z)+h)^{2}\right)^{3 / 2}},
$$

where $\sigma(z)$ is a stretching function. This stretching function has the property that $\sigma \ll 1$ for $0 \leq z<L_{j e t}$ where $L_{j e t}$ is the axial length of the jet and then at $z \simeq L_{j e t}$, the stretching function abruptly rises to unity so that $\sigma=1$ for $z>L_{j e t}$ consistent with there being no toroidal currents in the $z$ $>L_{j e t}$ region. The flux given by Eq. (17) is the same as that of Eq. (16) at $z=0$ but then is stretched out in the $z$ direction until $z \simeq L_{j e t}$ whereupon the flux given by Eq. (17) becomes the same as that of Eq. (16). Thus, $\psi$ is nearly independent of $z$ for $z<L_{j e t}$ and then suddenly decreases when $z \simeq L_{j e t}$. On a line where $r$ is constant and $z$ is varied, $\psi$ therefore has a plateau-like $z$-profile from $z=0$ up to the jet length $z \simeq L_{\text {jet }}$ at which location $\psi$ has a precipice-like steep decay. In the plateau region, $\partial \psi / \partial z$ will be near zero so there will be negligible $B_{r}$. At the precipice, both $\partial \psi / \partial z$ and $\partial^{2} \psi / \partial z^{2}$ will be large and negative corresponding to large positive $B_{r}$ and large positive $J_{\phi}$ and thus a large axial retarding force $-J_{\phi} B_{r}$.

\section{B. Main jet column}

In the main jet column, the jet velocity is mainly axial, i.e., $U_{z} \gg U_{r}$. We define $a(z)$ as the outer radius of the current channel, i.e., the poloidal current is finite in the region $0 \leq r \leq a(z)$ and zero immediately outside this region. The slight widening or flaring of the jet radius as $z$ increases means that the jet radius $a$ is a weakly increasing function of $z$, i.e., $a=a(z)$ as indicated in Fig. 3 where $a(z)$ is a weak function of $z$. Thus for $r^{2} \ll(z \sigma(z)+h)^{2}$ Eq. (17) could be expressed as $\psi \sim r^{2} /(z \sigma(z)+h)^{3}$ indicating $a(z)$ $=a(0)(1+z \sigma(z) / h)^{3 / 2}$. Thus, in the main jet column, i.e., in the region $0 \leq r<a(z)$ where the poloidal current is finite, the poloidal flux has the form
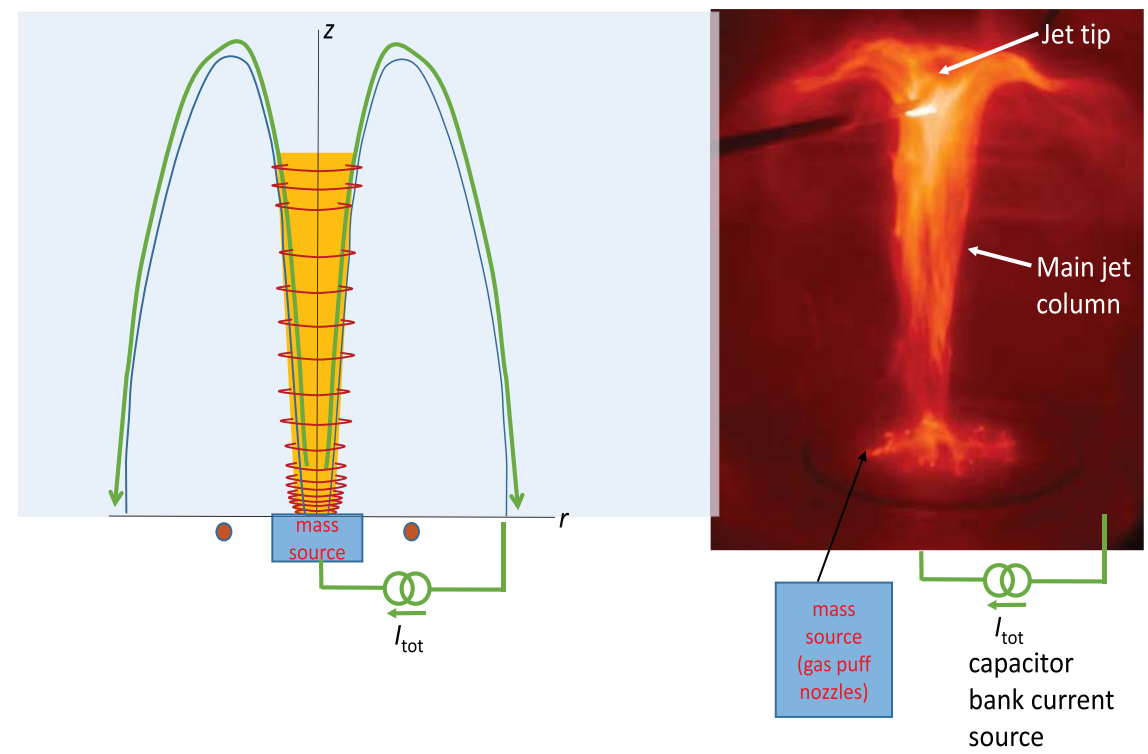

FIG. 4. Comparison between jet sketch and photo of lab jet. The dark rod at upper left in the photo is a magnetic probe. 


$$
\psi(r, z)=\psi_{0}\left(\frac{r}{a(z)}\right)^{2},
$$

which corresponds to an approximately radially uniform $B_{z}$ for $0 \leq r<a(z)$. Time-dependence has been suppressed because the main jet column is assumed to be quasi-steady. The weak $z$-dependence of $a(z)$ means that derivatives with respect to $z$ can be neglected when in competition with derivatives with respect to $r$, i.e., $\partial / \partial z \ll \partial / \partial r$. Thus, in the main jet column $\left|B_{r} / B_{z}\right|=\left|(\partial \psi / \partial r)^{-1} \partial \psi / \partial z\right| \sim r / z$ is small so the poloidal magnetic field is mainly in the $z$ direction. Furthermore, because $\psi \sim r^{2}$, the elliptic operator appearing in the equation of motion has the form

$$
r^{2} \nabla \cdot\left(\frac{1}{r^{2}} \nabla \psi\right)=r \frac{\partial}{\partial r}\left(\frac{1}{r} \frac{\partial \psi}{\partial r}\right)+\frac{\partial^{2} \psi}{\partial z^{2}}=\frac{\partial^{2} \psi}{\partial z^{2}} .
$$

This means that terms involving $r^{2} \nabla \cdot\left(r^{-2} \nabla \psi\right)$ are of order $\partial^{2} / \partial z^{2}$ and so can be neglected relative to terms having no or fewer $z$ derivatives.

\section{Radial component of the equation of motion}

Because $U_{r}$ is assumed small compared to $U_{z}, U_{r}$ will be dropped in Eq. (14a) so this equation reduces to

$$
\frac{\mu_{0} I}{4 \pi^{2} r^{2}} \frac{\partial I}{\partial r}=-\frac{\partial P}{\partial r} ;
$$

this is just the Bennett pinch relation $J_{z} B_{\phi}=-\partial P / \partial r$ of a straight column since $B_{\phi}=\mu_{0} I / 2 \pi r$ and $J_{z}=(2 \pi r)^{-1} \partial I / \partial r$. Equation (20) is essentially the statement that because the flaring is so weak, it can be ignored when computing radial forces. The result is a local radial force balance between the radially outward pushing of the pressure and the radial inward pushing of the magnetic pinch force. Even though there is compression of an axial magnetic field, little radial force is associated with this compression. This is mathematically the consequence of Eq. (19) and B being nearly straight. The poloidal magnetic field which is mainly a $B_{z}$ field exerts a much smaller associated radial magnetic force than the toroidal magnetic field despite the toroidal field being smaller than the poloidal magnetic field. This is because the toroidal field has a much larger radial gradient and a much smaller radius of curvature than does the poloidal magnetic field and Eq. (5) shows that gradient and curvature are important requirements for existence of a magnetic force.

The radial boundary condition for Eq. (20) is that $P=0$ at $r=a(z)$. In order to have the simplest non-trivial dependence of $I$ on $\psi$ it will be assumed that

$$
\mu_{0} I=\lambda \psi
$$

where $\mu_{0}$ has been inserted to make $\lambda$ have dimensions of inverse length as in spheromak literature. Using Eqs. (18) and (21) in Eq. (20) gives

$$
\frac{\partial P}{\partial r}=-\frac{\lambda^{2} \psi_{0}^{2}}{2 \pi^{2} \mu_{0} a^{4}} r .
$$

Integration and imposition of the boundary condition that $P(a(z))=0$ gives

$$
P(r, z)=\frac{\lambda^{2} \psi_{0}^{2}}{4 \pi^{2} \mu_{0} a(z)^{2}}\left(1-\frac{r^{2}}{a(z)^{2}}\right) .
$$

Equation (23) shows that $P$ is larger at axial locations where $a$ is smaller. This will provide an axial hydrodynamic force pushing from axial regions with small $a$ to axial regions with large $a$. This non-magnetic force peaks on the $z$-axis.

\section{Azimuthal component of the equation of motion}

We assume that $I=I(\psi)$ so $\nabla I \times \nabla \psi=0$ which implies there is no current flowing across poloidal flux surfaces in the upper half $r-z$ plane. Thus, all terms in Eq. (14b) vanish. Section VII shows that if $I$ is time-dependent as would happen when the current source is turned on, then $I$ $\neq I(\psi)$ and a transient poloidal current with zero timeaverage flows across poloidal flux surfaces. This current normal to poloidal flux surfaces produces a finite-duration, axially dependent torque which results in a transient axially dependent $U_{\phi}$ and so a finite twist.

\section{Axial component of the equation of motion}

Consideration of Eq. (14c) and taking into account Eq. (19) show that the term $\partial \psi / \partial z \nabla \cdot\left(r^{-2} \nabla \psi\right)$ in Eq. (14c) is third order in $\partial / \partial z$, whereas the other terms are first order in $\partial / \partial z$. Because a weak $z$ dependence is assumed, the term $\partial \psi / \partial z \nabla \cdot\left(r^{-2} \nabla \psi\right)$ will therefore be neglected with respect to the other terms. It was also assumed that $U_{r} \ll U_{z}$ so terms involving $U_{r}$ will be neglected. Assuming a quasisteady state (i.e., $\partial / \partial t$ small compared to other terms), Eq. (14c) reduces to

$$
\frac{\partial}{\partial z}\left(\rho U_{z}^{2}\right)=-\frac{\mu_{0} I}{4 \pi^{2} r^{2}} \frac{\partial I}{\partial z}-\frac{\partial P}{\partial z}
$$

which can be integrated with respect to $z$ to obtain

$$
\rho U_{z}^{2}+\frac{\mu_{0} I^{2}}{8 \pi^{2} r^{2}}+P=\text { const. }
$$

From Eqs. (18) and (21) it is seen that $I^{2} \sim r^{4}$ so the middle term in Eq. (25) vanishes on the $z$-axis. Thus, on the $z$-axis Eq. (25) reduces to the Bernoulli relation, i.e.,

$$
\rho U_{z}^{2}(z)+P(z)=\text { const. for } r=0 .
$$

This gives an estimate of the jet velocity at the jet tip assuming that the jet velocity is nearly zero at the jet base (i.e., at $z=0$ ). At large $z$, the jet radius is much larger than at the jet base and so Eq. (23) indicates that we can approximate $P$ to be near zero at the jet tip. Since $\mu_{0} I(a)=\psi_{0}$ evaluation of Eq. (26) at $z=0$ (jet base) and at the jet tip (large $z$ ) gives for the jet axis

$$
P(z=0)=\left[\rho U_{z}^{2}(z)\right]_{t i p}
$$


or

$$
\left[U_{z}^{2}(z)\right]_{t i p}=\frac{\mu_{0} I_{t o t}^{2}}{4 \pi^{2} a(0)^{2}[\rho]_{\text {tip }}}=\frac{B_{\phi}^{2}(a(0), 0)}{\mu_{0}^{2}[\rho]_{\text {tip }}}
$$

where $B_{\phi}(a(0), 0)$ is the azimuthal magnetic field at $r=a(0)$, $z=0$ and $I_{\text {tot }}$ is the total jet poloidal current, i.e., $I_{\text {tot }}$ $=I(a(z), z)=\lambda \psi_{0} / \mu_{0}$. Equation (28) shows that the axial velocity at the tip is proportional to the total poloidal current and scales in a manner similar to the Alfvén velocity. However, the magnetic field is not the field at the tip, but rather is the edge azimuthal magnetic field at the base.

The Bernoulli relation at $r=0$ given by Eq. (26) is a limiting form of a more general $r$-dependent expression. The $r$-dependence results because the accelerating force from $J_{r} B_{\phi}$ which is zero at $r=0$ becomes finite for finite $r$, while the axial pressure gradient decreases from its value at $r=0$ because of the radial dependence of pressure as given by Eq. (23). Substituting for $P$ from Eq. (23) and using $I=\mu_{0}^{-1} \psi_{0}(r / a(z))^{2}$, Eq. (25) becomes

$$
\rho U_{z}^{2}+\frac{\lambda^{2} \psi_{0}^{2}}{4 \pi^{2} \mu_{0} a(z)^{2}}\left(1-\frac{r^{2}}{2 a(z)^{2}}\right)=\mathrm{const},
$$

which causes the velocity to have a radial dependence. The $J_{r} B_{\phi}$ term can be expressed as

$$
J_{r} B_{\phi}=-\frac{\mu_{0} I}{4 \pi^{2} r^{2}} \frac{\partial I}{\partial z}=-\frac{\partial}{\partial z}\left(\frac{B_{\phi}^{2}}{2 \mu_{0}}\right)
$$

and so the off-axis axial acceleration results, in part, from the axial gradient of the energy density of the azimuthal magnetic field. This axial gradient results from the flaring of the jet main column. Equation (29) shows that the $J_{r} B_{\phi}$ term is the same order of magnitude as the $-\partial P / \partial z$ pressure gradient but the former is maximum off the $z$-axis, while the latter is maximum on the $z$-axis.

\section{Tip of the jet: Collimation}

\section{Qualitative analysis}

The assumption that $\partial / \partial z$ is small becomes untrue at the tip of the jet because here the poloidal field is curved with a small radius of curvature. The associated magnetic curvature force is directed to retard the jet so the velocity of the jet tip will be less than that of the main body. This implies existence of a bunching or stagnation at axial locations just below the tip. The situation is analogous to a group of cars going $80 \mathrm{~km} / \mathrm{h}$ on a highway with the cars far behind going $100 \mathrm{~km} / \mathrm{h}$ and having to slow down as they catch up with the $80 \mathrm{~km} / \mathrm{h}$ group. An observer moving at $90 \mathrm{~km} / \mathrm{h}$ between the two groups will see cars from behind approaching at $100 \mathrm{~km} / \mathrm{h}$ and cars in front at $80 \mathrm{~km} / \mathrm{h}$. In the observer's frame, it will appear that the cars behind are approaching at $10 \mathrm{~km} / \mathrm{h}$ and the cars in front are also approaching at $10 \mathrm{~km} / \mathrm{h}$ so the density of cars will increase in the vicinity of the observer.
Thus, the region just before the jet tip will have an increase in density because of the axial compression resulting from the bunching. Since toroidal magnetic flux is frozen into the plasma, this axial compression of the plasma will axially compress the frozen-in toroidal flux and so increase $B_{\phi}$ since $B_{\phi}$ is the toroidal flux density. However, because $B_{\phi}$ provides the radial inward pinch force, the axial plasma compression resulting from the converging velocity in the jet frame will enhance the pinch force. This enhancement will reduce the jet radius near the tip and so collimate the jet. The necessity for reducing the jet radius can also be seen to be required because $I_{\text {tot }}$ is fixed and Ampere's law implies $\mu_{0} I_{\text {tot }}=2 \pi a(z) B_{\phi}(a(z))$ so amplification of $B_{\phi}$ at constant $I_{\text {tot }}$ necessitates a reduction in $a(z)$.

\section{Quantitative analysis of collimation}

The axial magnetic force is

$$
F_{z}=J_{r} B_{\phi}-J_{\phi} B_{r}
$$

and since $J_{r}, B_{\phi}, J_{\phi}$, and $B_{r}$ are all positive, the $J_{r} B_{\phi}$ term is identified as giving a force that accelerates the jet, while the $J_{\phi} B_{r}$ term provides a retarding force that decelerates the jet. The axial dependence of the jet radius used in Eq. (18) could be expressed as

$$
a(z)=a_{0} \exp \left(\int_{0}^{z} \kappa\left(z^{\prime}\right) d z^{\prime}\right)
$$

where $\kappa(z)$ is small and nearly independent of $z$ in the main jet column but abruptly becomes large as the main column turns into the jet tip because at the tip the poloidal flux contour abruptly changes from being vertical to being horizontal. Insertion of Eq. (32) in Eq. (18) gives a gradually flaring poloidal flux $\psi(r, z)$ in the main jet column and then a sudden broadening at the jet tip. This change affects $J_{\phi}$ since

$$
\begin{aligned}
J_{\phi} & =-\frac{1}{2 \pi r \mu_{0}}\left[r \frac{\partial}{\partial r}\left(\frac{1}{r} \frac{\partial \psi}{\partial r}\right)+\frac{\partial^{2} \psi}{\partial z^{2}}\right] \\
& =\frac{r \psi_{0}}{\pi a_{0}^{2} \mu_{0}}\left(\frac{d \kappa}{d z}-2 \kappa^{2}\right) \exp \left(-2 \int_{0}^{z} \kappa\left(z^{\prime}\right) d z^{\prime}\right) .
\end{aligned}
$$

Since $\kappa(z)$ and $d \kappa / d z$ are both small in the jet main body, $J_{\phi}$ is negligible in the main body. In contrast, at the jet tip where $d \kappa / d z$ abruptly becomes large, Eq. (33) shows that there will be a localized large, finite positive $J_{\phi}$ if the change is so abrupt that $d \kappa / d z>2 \kappa^{2}$. Furthermore, since $\psi$ suddenly drops when $a(z)$ suddenly becomes large, $B_{r}$ $=-(2 \pi r)^{-1} \partial \psi / \partial z$ will be substantial at the jet tip. The axial magnetic force $J_{r} B_{\phi}-J_{\phi} B_{r}$ will then become negative, i.e., become a retarding force near the jet tip. This decelerates the jet near the tip so the tip will move slower than the main jet body. An observer moving in the jet frame and located just before the tip will observe a converging axial velocity since plasma in front of the observer (closer to the tip) will be moving slower than the observer, while plasma behind the observer will be moving faster than the observer. 
The relation between tip deceleration and collimation can be seen from the toroidal component of the induction equation. Dotting Eq. (2) with $\nabla \phi$ gives

$$
\begin{aligned}
\frac{\partial}{\partial t}\left(\frac{B_{\phi}}{r}\right) & =\nabla \cdot[(\mathbf{U} \times \mathbf{B}) \times \nabla \phi] \\
& =\nabla \cdot\left[\mathbf{B} \frac{U_{\phi}}{r}-\mathbf{U} \frac{B_{\phi}}{r}\right] \\
& =\mathbf{B} \cdot \nabla\left(\frac{U_{\phi}}{r}\right)-\frac{B_{\phi}}{r} \nabla \cdot \mathbf{U}-\mathbf{U} \cdot \nabla\left(\frac{B_{\phi}}{r}\right) .
\end{aligned}
$$

Since $U_{\phi}=0$ is assumed, this can be re-arranged as

$$
\frac{d}{d t}\left(\frac{B_{\phi}}{r}\right)=-\frac{B_{\phi}}{r} \nabla \cdot \mathbf{U}
$$

where $d / d t=\partial / \partial t+\mathbf{U} \cdot \nabla$ is the convective derivative, i.e., the time derivative experienced by an observer moving with the fluid. Equation (35) shows, as predicted by the highway traffic analog, that $B_{\phi}$ increases if there is a converging flow velocity, i.e., when $\nabla \cdot \mathbf{U}$ is negative.

The equation of continuity, Eq. (1e), can be written as

$$
\frac{d \rho}{d t}+\rho \nabla \cdot \mathbf{U}=0
$$

so eliminating $\nabla \cdot \mathbf{U}$ between Eqs. (35) and (36) gives

$$
\left(\frac{B_{\phi}}{r}\right)^{-1} \frac{d}{d t}\left(\frac{B_{\phi}}{r}\right)=\rho^{-1} \frac{d \rho}{d t},
$$

which can be integrated to give

$$
\frac{d}{d t}\left(\ln \frac{B_{\phi}}{\rho r}\right)=0
$$

so $B_{\phi} / \rho r$ is constant in the jet frame. This implies that if $\rho$ increases, $B_{\phi}$ must correspondingly increase and so confirms that axial compression of the jet increases $B_{\phi}$. Ampere's law shows that this also implies $I_{\text {tot }} / \rho a(z)^{2}$ is constant in the jet frame. Since $I_{t o t}$ is fixed, increasing $\rho$ requires that $a(z)$ decreases and so axial compression of the jet tends to collimate the jet. Once the jet is collimated, $\partial / \partial z$ vanishes so there is no axial acceleration and the jet in the collimated region moves ballistically.

\section{SOLAR CORONAL LOOPS}

The direction of jet acceleration does not depend on the sign of the electric current since the magnetic forces prescribed by Eq. (5) depend on $B^{2}$. A solar coronal loop is an arched, twisted magnetic flux tube. The twist implies existence of an electric current flowing along the flux tube axis. The locations where the two bases of the arch intersect the solar surface are called footpoints so the arch spans two footpoints and the twist means that there is an electric current flowing from one footpoint to the other along the arched loop. Should there be no current flowing in the flux tube, the magnetic field in the flux tube would be a vacuum field and so would be the result of currents flowing below the solar surface. Since these currents are below the solar surface, the magnetic fields produced by these currents would become weaker with increasing altitude above the solar surface. This leads one to expect that if the flux tube consists of a vacuum magnetic field, the apex of the arched flux tube should be bulged as in Fig. 5(a), i.e., the apex would have field lines that are more widely spaced from each other than the corresponding field line spacing near the footpoints. However, observations show that solar loops are not bulged but instead are highly collimated, i.e., the cross-section at the apex is only slightly larger than at the footpoints. ${ }^{48}$

It was proposed in Ref. 49 that this observed collimation could be explained as a corollary of the jet model given here. It is presumed that there is a slight increase of loop crosssection on moving away from the footpoint and that there is an electric current flowing from one footpoint to the other as sketched in Fig. 5(b). Since jet acceleration is independent of the current polarity, there will be upward jet flows from both footpoints and these oppositely moving flows will collide at the apex. The collision at the apex will provide axial compression and hence collimation. The frozen-in toroidal-like magnetic flux shown as circles linking the arched loop in Fig. 5(b) is carried along by the flow and collides at the apex thereby amplifying the flux density associated with these loops, i.e., increasing the toroidal-like field and its associated pinch force and so collimating the loop.

\section{ASSOCIATION OF TOROIDAL VELOCITY WITH TIME-DEPENDENT CURRENT}

We now return to the jet geometry sketched in Figs. 1-4 and the associated analysis. It has been assumed that the poloidal current $I_{t o t}$ is constant and that there is no toroidal velocity $U_{\phi}$. We now validate this assumption by showing that $U_{\phi}$ is proportional to the time derivative of $I$, i.e., $U_{\phi}$ $\sim \partial I / \partial t$ and, furthermore, that any change in $U_{\phi}$ requires current to flow across poloidal flux surfaces. This can be deduced from either the single particle guiding center approximation or from the MHD equations. The single particle argument is more intuitive and so will be given. For simplicity, the discussion will be in the context of an initially (a)

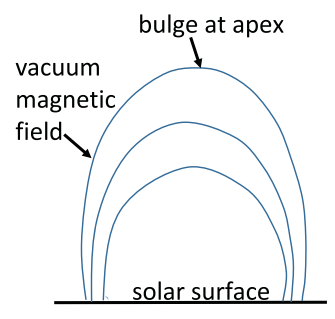

(b)

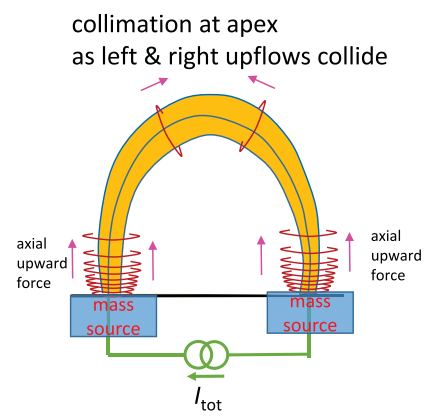

FIG. 5. (a) Arched vacuum solar magnetic field. (b) Arched solar magnetic field with current flowing along arch. Jet upflows are accelerated from both footpoints. These upflows collide at the apex, compressing the plasma and the frozen-in toroidal-like magnetic flux associated with the current. The amplified toroidal-like magnetic field collimates the flux tube which is now filled with plasma. 
straight, uniform cylindrical flux tube with no initial $B_{\phi}$ so the initial field is $\mathbf{B}=B_{z} \hat{z}$.

According to the guiding center approximation (Sec. 3.5.2 in Ref. 29), two types of particle drifts exist in a straight, uniform magnetic field, namely, the E cross B drift and the polarization drift. The E cross B drift is the same for electrons and ions and so can be written as the center of mass drift

$$
\mathbf{U}=\frac{\mathbf{E} \times \mathbf{B}}{B_{z}^{2}},
$$

which is equivalent to the ideal MHD Ohm's law [Eq. (1c)]. The polarization drift is

$$
\mathbf{v}_{p \sigma}=\frac{m_{\sigma}}{q_{\sigma} B^{2}} \frac{d \mathbf{E}_{\perp}}{d t},
$$

where $\perp$ means perpendicular with respect to the magnetic field. Summing the polarization drift over particles and species gives the polarization current

$$
\mathbf{J}_{p}=\sum_{\sigma=i, e} n q_{\sigma} \mathbf{v}_{p \sigma}=\frac{\rho}{B_{z}^{2}} \frac{d \mathbf{E}_{\perp}}{d t} .
$$

Crossing this current with $\mathbf{B}$ corresponds to

$$
\mathbf{J}_{p} \times \mathbf{B}=\rho \frac{d}{d t} \frac{\mathbf{E}_{\perp} \times \mathbf{B}}{B_{z}^{2}}=\rho \frac{d \mathbf{U}}{d t},
$$

which essentially retrieves the MHD equation of motion if $\nabla P$ is neglected. In this simplified model, the ideal Ohm's law prescribes $E_{z}=0$ so the azimuthal component of Faraday's law becomes

$$
\frac{\partial E_{r}}{\partial z}=-\frac{\partial B_{\phi}}{\partial t},
$$

which can be integrated with respect to $z$ to give

$$
E_{r}=-z \frac{\partial B_{\phi}}{\partial t} .
$$

Using Ampere's law $B_{\phi}=\mu_{0} I / 2 \pi r$, this becomes

$$
E_{r}=-z \frac{\mu_{0}}{2 \pi r} \frac{\partial I}{\partial t} .
$$

The picture of poloidal flux surfaces as being like insulated skins of an onion can be modified to be consistent with this point of view by imagining that the insulated onion skins are now separated from each other by a dielectric. The skins then act like the metal plates of a capacitor and so allow an $\mathrm{AC}$ current to flow between skins, but not a DC current. This impossibility of having a DC current flowing across flux surfaces in an ideal MHD plasma implies that the current source shown in the negative- $z$ region in Figs. 1-5 cannot be in a region governed by ideal MHD. ${ }^{50,51}$ For example, in constructing the numerical jet model in Ref. 52 it was found necessary to introduce below the $z=0$ plane a small non-MHD region where a fictitious spatially localized source term replaced the zero on the RHS of Eq. (2). This fictitious source term created toroidal magnetic field, i.e., created toroidal flux that could then move upwards.

The radial current density comes from Eq. (41) and is

$$
J_{r}=\frac{\rho}{B_{z}^{2}} \frac{\partial E_{r}}{\partial t}=-z \frac{\mu_{0}}{2 \pi r} \frac{\rho}{B_{z}^{2}} \frac{\partial^{2} I}{\partial t^{2}} .
$$

The toroidal velocity is given by Eq. (39) to be

$$
U_{\phi}=-\frac{E_{r}}{B_{z}}=z \frac{\mu_{0}}{2 \pi r B_{z}} \frac{\partial I}{\partial t}
$$

showing that $U_{\phi}=0$ if $I$ is constant. If the current is ramped from zero to a constant value, $\partial^{2} I / \partial t^{2}$ is initially positive, then drops to zero, while $\partial I / \partial t$ is finite and constant, and finally $\partial^{2} I / \partial t^{2}$ is negative until $\partial I / \partial t$ drops to zero. This can be understood in terms of the time dependence of $U_{\phi}$. The initial $\partial^{2} I / \partial t^{2}$ stage corresponds to acceleration in the $\phi$ direction $\left(U_{\phi}\right.$ increases from zero), the constant $\partial I / \partial t$ stage corresponds to constant $U_{\phi}$, and the negative $\partial^{2} I / \partial t^{2}$ stage corresponds to deceleration until $U_{\phi}$ is zero. The time integral of $U_{\phi} / r$ gives a finite angular displacement

$$
\Delta \phi=\int_{0}^{t} \frac{U_{\phi}}{r} d t=z \frac{\mu_{0} I}{2 \pi r^{2} B_{z}}=\frac{z}{r} \frac{B_{\phi}}{B_{z}} .
$$

Thus, the ramping up of $I$ to a finite value corresponds to a finite twisting of the plasma because $\Delta \phi$ is proportional to $z$. This analysis validates the assumption in Sec. V that $U_{\phi}=0$ when $I$ is constant. Reference 49 provides a similar proof using flux coordinates.

\section{EXPERIMENTAL AND NUMERICAL SUPPORT FOR THE MODEL}

Jets consistent with the concepts presented here have been studied in the author's laboratory at Caltech. The conditions that the ideal MHD description is a relevant description to an experiment depend on the characteristic scale length $L$ of the experiment and on plasma parameters. The conditions are: (i) the Lundquist number $S=\mu_{0} v_{A} L / \eta$ should be large compared to unity so that magnetic flux is frozen into the frame of the plasma and (ii) the ion skin depth $\delta_{i}=c / \omega_{p i}$ should be small compared to $L$ so that Hall terms can be neglected. The electron temperature in the Caltech experiments is $T_{e}>2 \mathrm{eV}$ so $\eta<2 \times 10^{-4} \mathrm{Ohm}-\mathrm{m}$ and a nominal Alfvén velocity is $v_{A}>10^{4} \mathrm{~m} \mathrm{~s}^{-1}$, while a nominal scale length is $L=0.2 \mathrm{~m}$ which gives $S>10$. The nominal density for an Ar plasma is $n=10^{22} \mathrm{~m}^{-3}$ corresponding to an ion skin depth $\delta_{i}=1.4 \times 10^{-2} \mathrm{~m}$ so $L>\delta_{i}$ is satisfied by the global jet motion. However, in certain circumstances ${ }^{53,54}$ the jet radial dimension can become comparable to an ion skin depth in which case Hall physics becomes important.

These experiments show collimation ${ }^{22,55}$ and scaling of jet velocity with current ${ }^{56}$ as predicted by Eq. (28). A $3 \mathrm{D}$ numerical MHD calculation ${ }^{52}$ with parameters corresponding to the Caltech experiment showed velocities and collimation consistent with the model predictions as well as verification of the Bernoulli-like relation. The numerical calculation was executed using dimensionless variables and so 
could be scaled both to the experiment and to an astrophysical jet situation.

A set of experiments simulating solar coronal loops has also been studied in the author's laboratory at Caltech and these have shown the predicted upflows from both footpoints. $^{25}$ Most recently, there has been a direct measurement $^{26}$ of the time-dependent vector magnetic field $\mathbf{B}(\mathbf{r}, t)$ in a $3 \mathrm{D}$ volume. This measurement had sufficient spatial resolution to allow for calculation of $\mathbf{J}$ from the curl of the measured magnetic field and thus calculation of the $3 \mathrm{D}$ magnetic force $\mathbf{J} \times \mathbf{B}$ in a $3 \mathrm{D}$ volume. The experimentally measured $\mathbf{B}$ and derived $\mathbf{J}$ demonstrated the predicted upward $\mathbf{J} \times \mathbf{B}$ force at both footpoints.

\section{ADDITIONAL FEATURES AND ASPECTS OF JETS}

Jets provide the context for a large variety of other phenomena which will only be briefly mentioned here since the details of these phenomena are complex and beyond the scope of this tutorial. These phenomena include:

1. Kinking of the jet. Because the axial magnetic field of a long jet is nearly in the $z$ direction, the combination of this field with the toroidal field implies that the jet magnetic field is nearly the same as the straight cylindrical approximation of a tokamak. The MHD kink instability involves a magnetically confined MHD plasma trying to coil up to release its magnetic energy. This tendency to coil up is because a coiled conductor has higher inductance than a straight conductor and because the magnetic energy of a flux conserving circuit scales inversely with its inductance (see Sec. 9.6 of Ref. 29). Periodic boundary conditions require kinking to satisfy the condition $\mathbf{k} \cdot \mathbf{B}$ $=2 \pi B_{z} / L+n B_{\phi} / a=0$ where $n$ is an integer; setting $n$ $=-1$ corresponds to the $q>1$ stability Kruskal-Shafranov kink stability condition relevant to tokamaks. Short jets effectively have $q \gg 1$ but as the jet lengthens, $q$ drops to unity at which point the jet kinks. This kinking has been studied in detail in experiments at Caltech ${ }^{24,57}$ and has also been observed in numerical simulations ${ }^{18,58}$ of astrophysical jets.

2. Rayleigh-Taylor instability. The exponentially growing helical deformation caused by the kinking means that the jet has a large acceleration perpendicular to the $z$ axis. Because the jet density exceeds that in the region outside the jet and because large lateral acceleration corresponds to the existence of a large effective gravity in the jet frame, the situation is one of a heavy fluid (i.e., the jet) on top of a light fluid (i.e., region outside the jet). A heavy fluid on top of a light fluid is subject to the RayleighTaylor instability wherein the heavy and light fluids interchange the average position via a rippling at the interface. For each downward heavy-fluid ripple, there is a corresponding adjacent upward light-fluid ripple. The gravitational potential energy of the system is thereby reduced and this liberation of potential energy goes into the kinetic energy of the ripples which thus become larger. This behavior was observed in the Caltech jet experiment where kinking spawned a distinct Rayleigh-Taylor instability ${ }^{53}$ having a faster time scale and a shorter length scale than the kink instability.

3. Breaking of the jet and reconnection. The RayleighTaylor instability occurs only on the trailing side of the laterally accelerating jet; this is because it is only on this side that the heavy fluid is on top of the light fluid. The consequence of this one-sidedness is that the jet current channel becomes choked as the low-density, light-fluid ripple eats into the jet cross-section. This choking of the jet radius increases the axial current density and it has been hypothesized ${ }^{53}$ that the increased axial current density can lead to an increase in local effective resistivity until there is essentially an open circuit. This behavior is observed to occur when the Rayleigh-Taylor ripples squeeze the jet width to be less than an ion skin depth and in this circumstance, ideal MHD is no longer an appropriate description as Hall terms must now be taken into account. This change in the magnetic structure corresponds to a breaking off of the jet and such a change in magnetic topology is a form of magnetic reconnection.

4. Collision of the jet with a target cloud. A jet might propagate in a low density background but then come to a region of high density. This will slow down the impacting jet and compress the density and magnetic flux in the jet. This process likely occurs with astrophysical jets that are presumed $^{59,60}$ to collide with molecular clouds; this process has been observed in laboratory jets. ${ }^{61}$

5. Shocks in the jet. The jet velocity depends on the mass density $\rho$ so if there is a modulation of the injected $\rho$ at the jet base, there will be an axial variation in the jet axial velocity. In this case, a faster axial segment of the jet will catch up with and collide with a slower moving segment and this will result in shock behavior. This is believed to occur in astrophysical jets and has been observed in the Imperial College laboratory experiment. ${ }^{62}$

6. Launching of the jet by an accretion disk. Steady injection of magnetic flux and mass at $z=0$ is inconsistent with the frozen-in flux properties of ideal MHD so it is necessary that the $z=0$ base be governed by equations beyond the scope of ideal MHD. This is important for modeling the coupling between accretion disks and astrophysical jets. An accretion disk has finite, negative $U_{r}$ and a portion of this radial inflow supplies the mass for the jet axial outflow. The radial component of Ohm's law [Eq. (1c)], namely, $E_{r}+U_{\phi} B_{z}-U_{z} B_{\phi}=0$, allows for finite $E_{r}$, but the azimuthal component of Eq. (1c) does not allow for finite $U_{r}$. The latter difficulty occurs because $B_{z}$ is assumed constant in the $z=0$ plane and the system is axisymmetric; this implies that $E_{\phi}=0$. Furthermore, because of combined axial symmetry and axisymmetry at the disk, $B_{r}$ must vanish in the disk and so the consequence is that there is no term to balance the $U_{r} B_{z}$ term in $E_{\phi}+U_{z} B_{r}-U_{r} B_{z}=0$. The author has proposed that the weak ionization of protostellar accretion disks shows that the ideal MHD Ohm's law should be replaced by a Hall Ohm's law. This replacement makes it possible to have the released gravitational potential energy of the accreting material generate a radial electric field that then drives jets. ${ }^{50,51}$ The model shows that an inward radial current in 
the accretion disk completes the electric circuit involving the jet poloidal current. This radial inward current is constant and flows across flux surfaces in the accretion disk and so is contrary to the constraints posed by the ideal MHD equations. The model showed that if a particle has a charge-to-mass ratio so small that the effective cyclotron frequency is of the order of the Kepler frequency, then certain charged particles, namely, those with zero canonical angular momentum, could move across flux surfaces and provide a constant radially inward current. It was proposed that these zero canonical angular momentum particles would not be actual particles but instead would be the center of mass of a clump of collisionally coupled neutrals and ions in the very weakly ionized plasma. The effective mass of the clump is that of the neutrals and the effective charge is that of the ions so the clump charge-to-mass ratio would be suitably small.

7. Relativistic jets. If the jet becomes relativistic, then the mass density $\rho$ must have the relativistic form $\gamma \rho$ where $\gamma=\left(1-U^{2} / c^{2}\right)^{-1 / 2}$ and there is an electric force as well as a magnetic force. ${ }^{63}$

\section{COMPARISON OF THE MODEL PRESENTED HERE TO MODELS IN THE ASTROPHYSICAL LITERATURE}

The astrophysical literature contains jet models of varying complexity. For example, Lynden-Bell ${ }^{64}$ has presented a relatively simple analytical model containing great many simplifying assumptions whereas Staff et al., ${ }^{58}$ Pudritz, Hardcastle, and Gabuzda (PHB), ${ }^{65}$ and Ferreira and Deguiran ${ }^{66}$ have presented more complex models with fewer assumptions. We will now provide a brief comparison of the model presented here to Refs. 64-66 which are to be understood to be representative of the wide range of astrophysical models for jets and accretion.

Because Lynden-Bell uses a different notation from that used here, care must be used in relating Lynden-Bell's equations to those presented here. Lynden-Bell uses $P$ instead of $\psi$ to denote poloidal flux; thus Lynden-Bell's Eq. (2) is in agreement with Eqs. (6)-(8) given here. However, in contrast to the model presented here, Lynden-Bell assumes a forcefree condition, i.e., $\mathbf{J} \times \mathbf{B}=0$ and claims in his Eq. (2) that this implies

$$
B_{\phi}=\frac{\mu_{0}}{2 \pi r} I(\psi),
$$

where the dependence of $I$ on $\psi$ is to be determined [in Lynden-Bell's notation, $\mu_{0} I(\psi)$ becomes $\left.\beta(P)\right]$. Because the assumption of a force-free equilibrium corresponds to there being no pressure gradient and no acceleration, Lynden-Bell is essentially setting up a force-free version (i.e., zero pressure gradient limit) of Eq. (15), the Grad-Shafranov equation, namely,

$$
\frac{1}{\mu_{0}} \nabla \cdot\left(\frac{1}{r^{2}} \nabla \psi\right)+\frac{\mu_{0} I}{r^{2}} \frac{\partial I}{\partial \psi}=0 .
$$

On making the replacements $\psi \rightarrow P$ and $\mu_{0} I(\psi) \rightarrow \beta(P)$, Eq. (50) above becomes Lynden-Bell's Eq. (3). This is the spheromak equilibrium equation (8) except that the boundary conditions used by Lynden-Bell are very different from the conventional spheromak boundary condition of a perfectly conducting wall on which $\psi$ is uniform. Lynden-Bell instead assumes that the jet is a cavity inside an external medium having pressure $p(z)$ and that at the interface $B^{2} / 2 \mu_{0}=p(z)$. This is different in substance from the model presented here because Lynden-Bell effectively assumes that the jet is a massless bubble of magnetic field immersed in a highpressure external medium with the interface self-adjusting so that at each location on the interface the outward pressure of the magnetic bubble balances the inward pressure from the external medium. There is thus no description of the mass density in the jet, of the mass flux into the jet, of the fluid velocity in the jet, or of pressure gradients in the jet. In contrast, the model presented here presumes that the jet has mass, a mass flux, a radial pressure gradient such that the jet hydrodynamic pressure is maximum on the jet axis and zero at the outer radius of the jet, and that the jet has an axial velocity gradient which balances an axial force as seen from balancing the terms in Eq. (14c). In effect, Lynden-Bell is setting $\mathbf{U}=0$ and pressure $P=0$ in Eq. (14) to make the jet a massless bubble. The acceleration that occurs in LyndenBell's analysis occurs when the front of the magnetic bubble pushes against an external medium having a pressure rapidly decreasing with increasing $z$; as the bubble top feels less opposing force, the top of bubble accelerates upwards and the bubble elongates. This is similar to bubbles originating from the ocean bottom expanding as they rise to the surface where the ambient pressure is lower than that at the bottom. The acceleration is not from magnetic forces acting on the jet mass as Lynden-Bell's jet has zero mass and the internal magnetic field is force-free.

Unlike Lynden-Bell, but like the model presented here, the model of Pudritz, Hardcastle, and Gabuzda (PHB) ${ }^{65}$ is not force-free and furthermore assumes the jet contains mass to be accelerated. The PHB model consists of some analytical relations and then a full numerical solution of the MHD equations. The analytical relations assume a quasistatic system and conclude that $\mathbf{U}_{p o l}$ must be parallel to $\mathbf{B}_{p o l}$. This is consistent with the model presented here for the main jet column where the magnetic field is also assumed quasi-static. However, the quasi-static assumption does not apply to the jet tip because an observer standing in the jet path would see a large $\partial \mathbf{B} / \partial t$ as the jet tip sweeps past the observer's location. The PHB model allows for launching with finite $U_{\phi}$ and then considers how $U_{\phi}$ evolves, whereas the model presented here has $U_{\phi}=0$. Finite $U_{\phi}$ could be added to the model presented here and would result in an outward centrifugal force in Eq. (14a) so less hydrodynamic pressure $P$ would be required to balance the radially inward force of the magnetic pinch force. The analytical portion of the PHB model argues that $B_{\phi} \gg B_{z}$ in which case there would be very little retarding force from stretching the poloidal field. In comparison, the model presented here does not assume $B_{\phi} \gg B_{z}$.

The model ${ }^{58}$ of Staff et al. uses the numerical MHD methods described in PHB to model actual astrophysical jets observed by the Hubble Space Telescope. The resulting 
models are quite similar to the Caltech jet experiment, the main exception being that the kinking in the model of Staff et al. has more periods of kinking and the kink amplitude saturates without destroying the jet. The similarities can be seen by comparing Figs. 2-4 of Staff et al. to Fig. 4 here. Both the figures of Staff et al. and Fig. 4 here show a collimated jet that slows down (stagnates) near the jet tip (see Fig. 4 of Staff $e t a l$.) and has an increase in density near the tip as a result of this stagnation (see Fig. 3 of Staff et al.). This slowing and associated axial compression of the density is consistent with the discussion in Sec. V C here.

Ferreira and Deguiran ${ }^{66}$ consider what is needed for disks to launch jets and conclude that jets cannot be launched from disks unless there is some anomalous turbulent viscosity in the disk. Their analysis is based on self-similar models and so does not take into account the complete poloidal current circuit nor the location of the toroidal current distribution responsible for creating the poloidal flux. The jet launching is a complicated process according to Ferreira and Deguiran and cannot be described by a few simple equations. As discussed in item 6 of Sec. IX, the author has proposed a Hall MHD, weak disk ionization mode ${ }^{50,51}$ where no anomalous viscosity is required to launch a jet from a disk. Instead, a current source results from inward spiraling clumps of neutrals that are collisionally bound to a small number of ions. This inward motion of mass and positive charge corresponds to accretion and to radial electric current while the accumulation of positive charge at small radius produces a radially outward electric field that both drives the jet current and injects toroidal flux into the jet. This model provides boundary conditions essentially identical to those of the Caltech experiment and the experiment shows very clearly that imposition of such boundary conditions causes jets to be launched.

\section{SUMMARY}

MHD-driven jets are shown to have different mechanisms dominating in different parts of the jet. This can be understood in terms of the competition between the forces resulting from magnetic field curvature and from magnetic field gradients. The jet main column is squeezed by the pinching resulting from the toroidal magnetic field and is accelerated on axis by the gradient of the axial pressure gradient resulting from the axial gradient of this squeezing. Offaxis magnetic forces resulting from the axial gradient of the toroidal field provide axial acceleration. The jet tip has a retarding force because of the strong curvature of the poloidal magnetic field at the apex. This results in an axial nonuniformity of the jet velocity such that the tip velocity moves more slowly than the fluid behind the tip. The stagnation in the jet frame resulting from this axial non-uniformity compresses embedded toroidal magnetic flux, thereby amplifying the frozen-in toroidal magnetic field and so enhancing the pinching to produce collimation of the jet. Experimental measurements support these arguments. Because the ideal MHD equations have no intrinsic scale, these conclusions are relevant to solar and astrophysical plasmas as well as lab plasmas.

\section{ACKNOWLEDGMENTS}

This material was based on work supported by the U.S. Department of Energy Office of Science, Office of Fusion Energy Sciences under Award No. DE-FG02-04ER54755, by the National Science Foundation under Award No. 1348393, by the Air Force Office of Scientific Research under Award No. FA9550-11-1-0184, and by the U.S. Department of Energy Advance Research Projects Agency Energy under Award No. DE-AR0000565.

${ }^{1}$ L. Mestel, Mon. Not. R. Astron. Soc. 122, 473 (1961).

${ }^{2}$ A. Ferrari, Annu. Rev. Astron. Astrophys. 36, 539 (1998).

${ }^{3}$ R. D. Blandford and D. G. Payne, Mon. Not. R. Astronom. Soc. 199, 883 (1982).

${ }^{4}$ H. L. Marshall, B. P. Miller, D. S. Davis, E. S. Perlman, M. Wise, C. R. Canizares, and D. E. Harris, Astrophys. J. 564, 683 (2002).

${ }^{5}$ J. Chen and J. Krall, J. Geophys. Res.-Space Phys. 108, 1410, https:// doi.org/10.1029/2003JA009849 (2003).

${ }^{6}$ T. R. Jarboe, Plasma Phys. Controlled Fusion 36, 945 (1994).

${ }^{7}$ M. G. Rusbridge, S. J. Gee, P. K. Browning, G. Cunningham, R. C. Duck, A. alKarkhy, R. Martin, and J. W. Bradley, Plasma Phys. Controlled Fusion 39, 683 (1997).

${ }^{8}$ P. M. Bellan, Spheromaks: A Practical Application of Magnetohydrodynamic Dynamos and Plasma Self-Organization (Imperial College Press, London, 2000).

${ }^{9}$ J. Marshall, Phys. Fluids 3, 134 (1960).

${ }^{10}$ H. Maecker, Z. Fur Phys. 141, 198 (1955).

${ }^{11}$ M. Irie and M. R. Barrault, J. Phys. D-Appl. Phys. 10, 1599 (1977).

${ }^{12}$ H. Curtis, Publ. Lick Obs. 13, 31 (1918).

${ }^{13}$ E. M. D. Dal Pino, in Fundamentals of Space Environment Science, Advances in Space Research-Series Vol. 35, edited by V. Jatenco Pereira, A. C. L. Chian, J. F. Valdes Galicia, and M. A. Shea (Elsevier, Amsterdam, 2005), pp. 908-924; 1st Latin-American Advanced School on Space Environment, 22-27 March 2004, National Institute Space Research, Sao Jose dos Campos, Brazil.

${ }^{14}$ K. Shibata and Y. Uchida, Sol. Phys. 103, 299 (1986).

${ }^{15}$ R. V. E. Lovelace, H. L. Berk, and J. Contopoulos, Astrophys. J. 379, 696 (1991).

${ }^{16}$ R. Ouyed and R. E. Pudritz, Astrophys. J. 482, 712 (1997).

${ }^{17}$ J. M. Stone and P. E. Hardee, Astrophys. J. 540, 192 (2000).

${ }^{18}$ M. Nakamura, D. Garofalo, and D. L. Meier, Astrophys. J. 721, 1783 (2010).

${ }^{19}$ D. Lynden-Bell, Mon. Not. R. Astron. Soc. 341, 1360 (2003).

${ }^{20}$ S. V. Lebedev, A. Ciardi, D. J. Ampleford, S. N. Bland, S. C. Bott, J. P. Chittenden, G. N. Hall, J. Rapley, C. A. Jennings, A. Frank et al., Mon. Not. R. Astron. Soc. 361, 97 (2005).

${ }^{21}$ C. K. Li, P. Tzeferacos, D. Lamb, G. Gregori, P. A. Norreys, M. J. Rosenberg, R. K. Follett, D. H. Froula, M. Koenig, F. H. Seguin et al., Nat. Commun. 7, 13081 (2016).

${ }^{22}$ S. You, G. S. Yun, and P. M. Bellan, Phys. Rev. Lett. 95, 045002 (2005).

${ }^{23}$ P. M. Bellan, D. Kumar, E. V. Stenson, S. K. P. Tripathi, G. S. Yun, and A. L. Moser, "Plasmas in the laboratory and in the universe: Interactions, patterns, and turbulence," AIP Conf. Proc. 1242, 156-163 (2010).

${ }^{24}$ S. C. Hsu and P. M. Bellan, Phys. Plasmas 12, 032103 (2005).

${ }^{25}$ E. V. Stenson and P. M. Bellan, Phys. Rev. Lett. 109, 075001 (2012)

${ }^{26}$ M. A. Haw and P. M. Bellan, Geophys. Res. Lett. 44, 9525, https:// doi.org/10.1002/2017GL074505 (2017).

${ }^{27}$ M. Zuin, R. Cavazzana, E. Martines, G. Serianni, V. Antoni, M. Bagatin, M. Andrenucci, F. Paganucci, and P. Rossetti, Phys. Plasmas 11, 4761 (2004).

${ }^{28}$ D. R. Lev and E. Y. Choueiri, J. Propul. Power 28, 609 (2012).

${ }^{29}$ P. M. Bellan, Fundamentals of Plasma Physics (Cambridge University Press, Cambridge, 2006).

${ }^{30}$ P. M. Bellan, Magnetic Helicity, Spheromomaks, Solar Coronal Loops, and Astrophysical Jets (Imperial College Press, London, 2017).

${ }^{31}$ D. D. Ryutov, B. A. Remington, H. F. Robey, and R. P. Drake, Phys. Plasmas 8, 1804 (2001).

${ }^{32}$ M. D. Altschuler and G. Newkirk, Sol. Phys. 9, 131 (1969).

${ }^{33}$ T. Wiegelmann, G. J. D. Petrie, and P. Riley, Space Sci. Rev. 210, 249 (2017).

${ }^{34}$ L. Woltjer, Proc. Natl. Acad. Sci. U. S. A. 44, 833 (1958). 
${ }^{35}$ L. Woltjer, Proc. Natl. Acad. Sci. U. S. A. 44, 489 (1958).

${ }^{36}$ J. B. Taylor, Phys. Rev. Lett. 33, 1139 (1974).

${ }^{37}$ J. B. Taylor, Rev. Mod. Phys. 58, 741 (1986).

${ }^{38}$ S. Lundquist, Ark. Fys. 2, 361 (1950).

${ }^{39}$ J. Heyvaerts and E. R. Priest, Astron. Astrophys. 137, 63 (1984).

${ }^{40}$ G. E. Vekstein, E. R. Priest, and C. D. C. Steele, Sol. Phys. 131, 297 (1991).

${ }^{41}$ B. C. Low, Rev. Geophys. 20, 145, https://doi.org/10.1029/ RG020i001p00145 (1982).

${ }^{42}$ W. H. Bennett, Phys. Rev. 45, 890 (1934).

${ }^{43} \mathrm{H}$. Grad and H. Rubin, in Proceedings of the 2nd United Nations Conference on the Peaceful uses of Atomic Energy (IAEA, Vienna, 1958), Vol. 31, p. 190.

${ }^{44}$ V. D. Shafranov, Reviews of Plasma Physics (Consultants Bureau, New York, 1966), Vol. 2, p. 103.

${ }^{45}$ J. Wesson, Tokamaks (Clarendon Press, Oxford, 2004).

${ }^{46}$ A. Isavnin, E. K. J. Kilpua, and H. E. J. Koskinen, Sol. Phys. 273, 205 (2011).

${ }^{47}$ H. R. Lewis and P. M. Bellan, J. Math. Phys. 31, 2592 (1990).

${ }^{48}$ J. A. Klimchuk, Sol. Phys. 193, 53 (2000).

${ }^{49}$ P. M. Bellan, Phys. Plasmas 10, 1999 (2003).

${ }^{50}$ P. M. Bellan, Mon. Not. R. Astron. Soc. 458, 4400 (2016).
${ }^{51}$ P. M. Bellan, Plasma Phys. Controlled Fusion 60, 014006 (2018).

${ }^{52}$ X. Zhai, H. Li, P. M. Bellan, and S. T. Li, Astrophys. J. 791, 40 (2014).

${ }^{53}$ A. L. Moser and P. M. Bellan, Nature 482, 379 (2012).

${ }^{54}$ K. B. Chai, X. Zhai, and P. M. Bellan, Phys. Plasmas 23, 132122 (2016).

${ }^{55}$ G. S. Yun and P. M. Bellan, Phys. Plasmas 17, 062108 (2010).

${ }^{56}$ D. Kumar and P. M. Bellan, Phys. Rev. Lett. 103, 105003 (2009).

${ }^{57}$ S. C. Hsu and P. M. Bellan, Phys. Rev. Lett. 90, 215002 (2003).

${ }^{58}$ J. E. Staff, B. P. Niebergal, R. Ouyed, R. E. Pudritz, and K. Cai, Astrophys. J. 722, 1325 (2010).

${ }^{59}$ S. W. Higgins, T. J. O’Brien, and J. S. Dunlop, Mon. Not. R. Astron. Soc. 309, 273 (1999).

${ }^{60}$ Z. Wang, P. J. Wiita, and J. S. Hooda, Astrophys. J. 534, 201 (2000).

${ }^{61}$ A. L. Moser and P. M. Bellan, Astrophys. Space Sci. 337, 593 (2012).

${ }^{62}$ F. Suzuki-Vidal, S. V. Lebedev, S. N. Bland, G. N. Hall, G. Swadling, A. J. Harvey-Thompson, J. P. Chittenden, A. Marocchino, A. Ciardi, A. Frank et al., Phys. Plasmas 17, 112708 (2010).

${ }^{63}$ J. Contopoulos, Astrophys. J. 432, 508 (1994).

${ }^{64}$ D. Lynden-Bell, Mon. Not. R. Astron. Soc. 369, 1167 (2006).

${ }^{65}$ R. E. Pudritz, M. J. Hardcastle, and D. C. Gabuzda, Space Sci. Rev. 169, 27 (2012).

${ }^{66}$ J. Ferreira and R. Deguiran, High Energy Density Phys. 9, 67 (2013). 